\title{
FOXA1 mutations in hormone-dependent cancers
}

\author{
Jessica L. L. Robinson ${ }^{1,2}$, Kelly A. Holmes ${ }^{1}$ and Jason S. Carroll ${ }^{1,2 *}$ \\ ${ }^{1}$ Cancer Research UK, Robinson Way, Cambridge, UK \\ ${ }^{2}$ Department of Oncology, University of Cambridge, Cambridge, UK
}

Edited by:

Eric W. Lam, Imperial College

London, UK

Reviewed by:

Bernd Groner, Georg Speyer Haus,

Institut for Biomedical Research,

Germany

Enrico V. Avvedimento, University

Naples "Federico II", Italy

*Correspondence:

Jason S. Carroll, Cancer Research

UK, Robinson Way, Cambridge,

CB2 ORE, UK.

e-mail: jason.carroll@cruk.cam.ac.uk
The forkhead protein, FOXA1, is a critical interacting partner of the nuclear hormone receptors, oestrogen receptor- $\alpha(E R)$ and androgen receptor (AR), which are major drivers of the two most common cancers, namely breast and prostate cancer. Over the past few years, progress has been made in our understanding of how FOXA1 influences nuclear receptor function, with both common and distinct roles in the regulation of ER or AR. Recently, another level of regulation has been described, with the discovery that FOXA1 is mutated in $1.8 \%$ of breast and $3-5 \%$ prostate cancers. In addition, a subset of both cancer types exhibit amplification of the genomic region encompassing the FOXA1 gene. Furthermore, there is evidence of somatic changes that influence the DNA sequence under FOXA1 binding regions, which may indirectly influence FOXA1-mediated regulation of $E R$ and AR activity. These recent observations provide insight into the heterogeneity observed in ER and AR driven cancers.

Keywords: FoxA1, breast cancer, prostate cancer, exome sequencing, SNP, androgen receptor (AR), estrogen receptor alpha
Hormone-dependent breast and prostate cancers, constitute a major global cancer burden, in females and males, respectively, with a combined total of nearly 2.3 million new cases each year (Ferlay et al., 2010). In a large proportion of these cancers, the nuclear receptors AR in prostate cancer and ER in breast cancer, drive tumor growth in response to activation by their natural ligands, testosterone and oestrogen. A protein commonly expressed in hormonally-driven cancers is the transcription factor FOXA1 which appears to be intrinsic to tumor development in both breast and prostate cancer. Recent discoveries of amplification of the FOXA1 locus, mutations within the FOXA1 gene and mutations in the genomic regions FOXA1 occupies, are shedding light on mechanisms that perturb FOXA1 function and ultimately ER/AR activity.

\section{FOXA1 IS CRITICAL FOR AR AND ER FUNCTION IN CANCER}

FOXA1 is one of three members of the highly related FOXA family. All forkhead proteins contain a "winged helix" DNA binding or forkhead domain, consisting of three $\alpha$-helices, three $\beta$-sheets, and two loops or wings (Hannenhalli and Kaestner, 2009). The crystal structure of the forkhead domain shows that FOXA1 sits in the major groove of DNA with the loops making site-specific DNA contacts in a manner that closely resembles linker histone (Clark et al., 1993). FOXA proteins are often termed "pioneer factors" because they are able to bind to highly compacted, or "closed," chromatin (Cirillo et al., 1998) and through their C-terminal domain make these genomic regions more accessible to other transcription factors (Cirillo et al., 2002) (Figure 1A).

Like other forkhead proteins, FOXA1 plays a key role in development, chiefly the lung and liver (Wan et al., 2004; Lee et al., 2005). With regards to the development of mammary glands and prostate rudiment, the FoxA1 ${ }^{-/-}$mouse shows a normal phenotype at birth, but neither tissue is able to respond to hormone induction through AR and ER during puberty which results in ductal branching and epithelial cell maturation (Gao et al., 2005; Bernardo et al., 2010). FOXA1's direct interaction with AR and ER was first shown at the single locus level, probasin and PSA for AR (Gao et al., 2003), and vitellogenin for ER (Robyr et al., 2000). Later genome wide chromatin-immunoprecipitation experiments (ChIP-seq) for AR, ER, and FOXAl in breast and prostate cancer cell lines and primary tumor tissue revealed a high level of co-occupancy between this pioneer factor and its respective nuclear receptor, presumably mediated by the forkhead motif found at AR and ER binding events (Carroll et al., 2006; Wang et al., 2007; Jia et al., 2008; Hurtado et al., 2011). Loss of FOXA1 expression by targeted siRNA transfection in breast and prostate cancer cell lines results in reduction of growth suggesting an essential role in the proliferation of both cancers (Hurtado et al., 2011; Robinson et al., 2011; Zhang et al., 2011) yet the mechanistic role for FOXA1 in ER and AR biology appears to be different.

FOXA1 is required for global ER binding in the MCF7 breast cancer cell line and ER occupancy at $>90 \%$ of binding events is reduced when FOXA1 is silenced, which correlates with a global loss in the accessibility of chromatin (Hurtado et al., 2011). This loss of ER binding blocks ER-mediated gene expression and proliferation. In prostate cancer, however, when FOXA1 is lost, AR can still bind to chromatin and in fact occupies new binding sites which are coupled to changes in its' transcriptional targets (Sahu et al., 2011; Wang et al., 2011). Moreover, FOXA1 expression levels have opposing effects on patient outcome in breast and prostate cancer. In breast cancer, FOXA1 has been shown in multiple studies to be an independent marker for good outcome 


\title{
A FOXA1 opens 'closed' chromatin to facilitate AR and ER binding
}
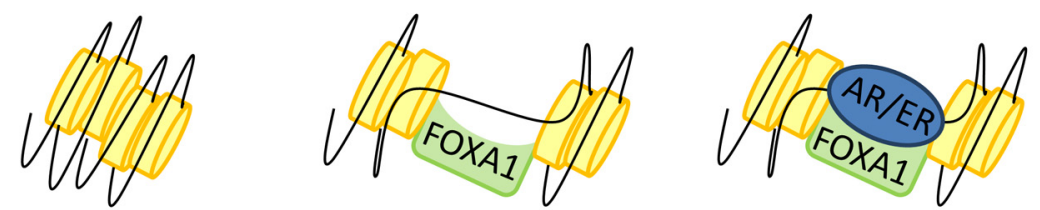

\section{B Consequences FOXA1 genomic alterations could have on AR and ER binding in breast and prostate cancer}

\author{
Breast-Greater \\ dependence upon $E R$ \\ for growth, so \\ hormonal therapies \\ are more effective.
}

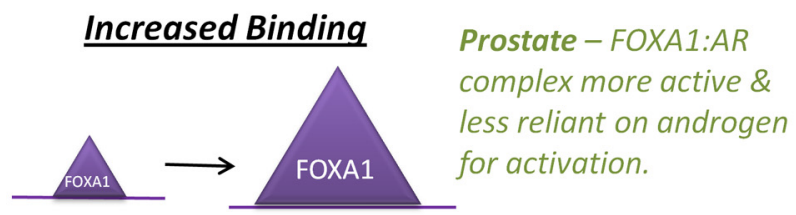
Breast - ER directed to new regions resulted in differential gene expression program

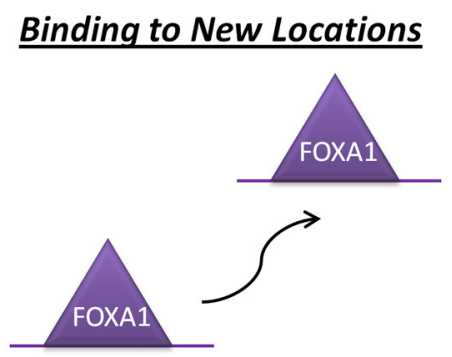

$$
\begin{aligned}
& \text { Prostate-AR directed to } \\
& \text { new regions resulted in } \\
& \text { differential gene } \\
& \text { expression program. } \\
& \text { FOXA1 recruits other } \\
& \text { transcription factors. }
\end{aligned}
$$

Breast - ER unable to bind so hormonal therapies ineffective

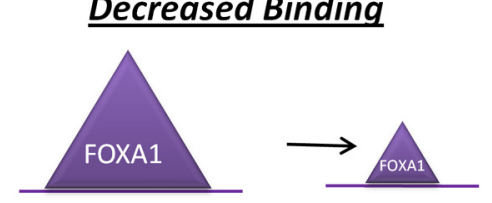

\begin{abstract}
Prostate - AR stills
binds but expresses

different genes
\end{abstract}

FIGURE 1 | Interactions between FOXA1 and the hormone receptors AR and ER. (A) WT FOXA1 is able to bind to condensed chromatin and make this region more accessible to $A R$ and $E R$ which bind to DNA after hormone stimulation. (B) Various genomic alterations can occur to FOXA1 such as amplification of the FOXA1 locus, mutations to the coding sequence of FOXA1 or single nucleotide variants (SNVs) in the forkhead motif found at most FOXA1 binding events. This could results in an increase, decrease, or change in position of FOXA1 binding which in turn could affect $A R$ and ER in the ways described above.
(Badve et al., 2007; Hisamatsu et al., 2012); most probably because the presence of FOXA1 indicates a functional ER complex which will respond well to anti-oestrogen compounds such as Tamoxifen. Conversely, in prostate cancer, high levels of FOXA1 correlate with poor prognosis (Sahu et al., 2011; Gerhardt et al., 2012). FOXA1 and AR levels correlate with one another, suggesting that an overactive AR transcriptional complex may be present in these tumors to overcome androgen deprivation more effectively. Interestingly, there is evidence of a common reliance in late stage disease as FOXA1 levels are high in both breast and prostate cancer metastases (Jain et al., 2011; Ross-Innes et al., 2012).

\section{ADVANCES IN DNA SEQUENCING TECHNOLOGIES}

Genomic sequencing capacity has increased at an extraordinary rate, enhancing our ability to interrogate genetic changes and global genomic patterns. Since the first cancer genome was sequenced in 2008 (Ley et al., 2008), there have been numerous other sequencing studies with increasing numbers of tumors at greater genome coverage, made possible by improvements in DNA sequencing output coupled with a large decrease in cost (Meyerson et al., 2010). Whole exome- and whole-genome sequencing allow unbiased sequencing of cancer vs. normal exons or entire genomes to look for cancer specific somatic mutations and chromosomal rearrangements, amplifications or 
deletions. All the studies in this review have a minimum of 30 -fold coverage over each gene, meaning it is possible to detect mutations which may only occur in a subset of cells in the tumor. Transcriptomic data from RNA-seq is also being mined to assess for changes in transcript expression levels that are influenced by these mutations. Integration of these genetic alterations will provide insight into the events that contribute to tumor progression.

\section{MUTATIONS IN PROSTATE CANCER}

Unlike breast cancer, prostate cancer patients cannot be prognostically stratified based on mRNA expression profiles, therefore it is critical that genomic contributors to outcome are defined. Two large prostate cancer sequencing papers recently reported that FOXA1 mutations occur in 3.4-5.2\% of tumors (Barbieri et al., 2012; Grasso et al., 2012). This finding has not been observed in any of the previous prostate cancer sequencing studies, probably because those earlier studies either lacked the number of samples or the sequencing depth required to detect variants that were present at low frequency (Taylor et al., 2010; Berger et al., 2011; Kumar et al., 2011).

The first study to report mutations in FOXA1 performed whole exome sequencing on 112 treatment-naive prostate adenocarcinomas as well as RNA-seq analysis on transcripts from 63 tumors (Barbieri et al., 2012). They found 12 genes which were significantly mutated, all of which were highly expressed in prostate cancer. A total of eight non-silent mutations in FOXA1 were identified clustered around the forkhead DNA binding domain (Table 1). Grasso et al., independently discovered by exome sequencing, FOXA1 to be mutated in a single tumor from their cohort of 11 high-grade localized prostate cancers but in none of their 50 metastatic castrate resistant prostate cancers (CRPC) obtained from rapid autopsies (Grasso et al., 2012). The mutation was a 2 bp insertion which resulted in a frame shift (S453fs) and two more frame shift mutations were detected in FOXA1 from exome sequencing of 11 prostate cancer cell lines (P358fs in hormone-sensitive LAPC-4 and A339fs in castrate resistance model DU-145). Based on this initial observation, a total of 101 localized prostate cancers and 46 CRPCs were subsequently targeted sequenced, within which five harbored mutations in FOXA1. Only one CRPC sample harbored a FOXA1 mutation which was isolated from a patient included in the initial exome study, but the DNA used in the targeted sequencing was obtained from a different site suggesting the FOXA1 mutation was a divergent event not seen in the primary tumor.

\section{MUTATIONS IN BREAST CANCER}

Six major breast cancer genomic studies were published in 2012 which utilize high-throughput sequencing techniques to tease

Table 1 | FOXA1 mutations in breast and prostate cancer.

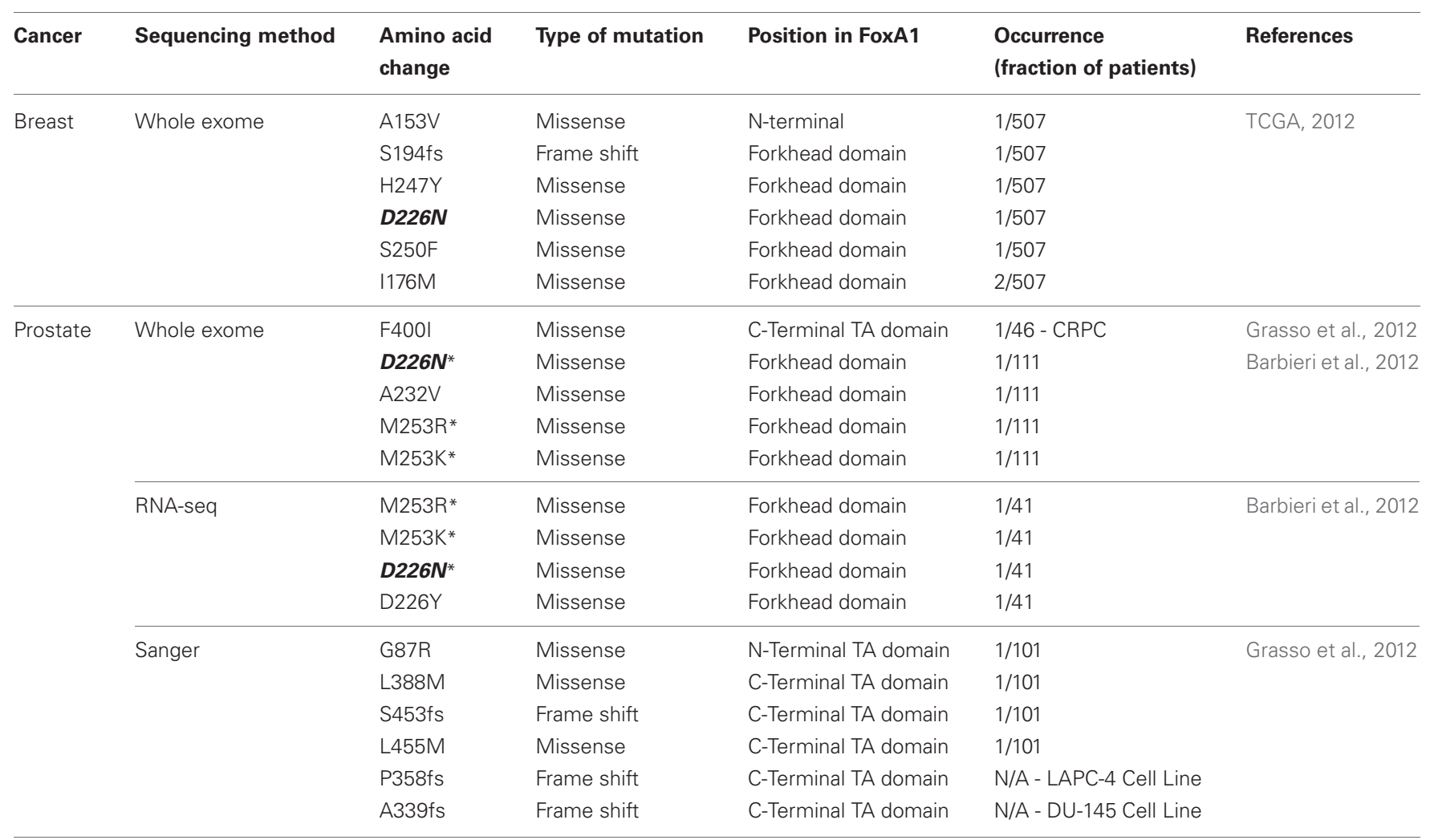

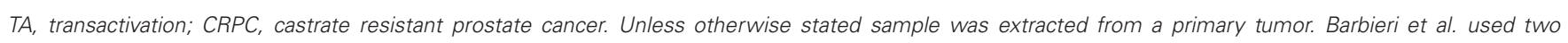

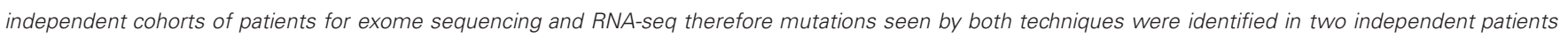
(indicated by *). D226N is the only mutation seen in both breast and prostate cancer. 
apart the important mutations, chromosomal rearrangements, and DNA methylation changes which occur in breast cancer. The findings have reinforced the role of known breast cancer driver genes such as TP53, ERBB2, (HER2) and PIK3CA and led to the discovery of a plethora of novel oncogenic mutations, including FOXA1 in The Cancer Genome Atlas (TCGA) study (TCGA, 2012). The TCGA study assessed a total of 825 patients to ranging extents on six different platforms, including exome sequencing of 507 tumors and matched normal DNA. They identified eight tumors with mutations in FOXA1 (Table 1) placing it on the cusp of being a significantly mutated gene as determined by the MuSiC package (Dees et al., 2012). In total, they found 35 genes significantly mutated and two genes at borderline significance (FOXA1 and CTCF). This raises an important point because none of the other four studies which carried out whole-exome or whole-genome sequencing reported any FOXA1 mutations (Banerji et al., 2012; Ellis et al., 2012; Shah et al., 2012; Stephens et al., 2012). These studies all had smaller sample sizes (ranging from 77 to 108 samples), which would not have been sufficient to detect FOXA1 mutants which, based on the TCGA study, only occur in 1.8\% patients. Furthermore, one of the studies (Shah et al., 2012) used a selected population of exclusively ER-PR-HER2-patients and all FOXA1 mutations in the TCGA study were found in ER+ tumors.

Gene expression profiling was applied to the eight tumors identified with FOXA1 mutations and the majority were luminal A tumors (5/8) and the remainder were luminal B (2/8) and HER2 (1/8). Another ER interacting partner, GATA3, was found to be highly mutated in all the studies $(10.8 \%$ of breast cancers in TCGA study), although GATA3 and FOXA1 mutations were mutually exclusive.

\section{F0XA1 COPY NUMBER ALTERATIONS}

The genomic region encompassing the FOXA1 gene (14q21.1) is amplified in a range of cancers (Yasui et al., 2001; Lin et al., 2002; Nucera et al., 2009; Deutsch et al., 2012) and there is increasing evidence this also occurs in prostate and breast cancer. FOXA1 amplification in prostate cancer was identified from the profiling of eight systemic metastatic tumors in a range of organs from six unrelated patients. Focal amplification of $14 \mathrm{q} 21.1$ was found in three independent lesions isolated from a single patient, suggesting amplification of the FOXA1 genomic region is an early event in progression of this tumor (Robbins et al., 2011). Furthermore, there is evidence of amplification of FOXA1 in a lymph node metastasis in an independent cohort (Grasso et al., 2012).

In breast cancer, the TCGA study reported that $1 \%$ of the 773 breast cancer tumors tested have focal amplification of the region containing the FOXA1 genomic locus, consisting of six ER+ and two HER2 tumors (TCGA, 2012). However, there was no evidence of amplification of this region in the larger study published by Curtis et al. which categorized 1992 breast cancers on the basis of copy number changes and mRNA profiles (Curtis et al., 2012) even though this study generally correlates well with the TCGA data.

\section{SEQUENCE CHANGES OF THE DNA BINDING MOTIF MODULATE FOXA1 BINDING}

FOXA1 binds primarily at enhancer regions of genes throughout the genome most of which contain the consensus forkhead (FKH) motif. Recently, a report described that single nucleotide polymorphisms (SNPs) in the binding sites of forkhead proteins can not only modulate FOXA1 binding to the chromatin, but lead to changes in expression of adjacent genes (Cowper-Sal Lari et al., 2012). Cowper-Sal Lari et al. developed a novel computational method called intragenomic replicates (IGR) to accurately predict the affinity of a transcription factor for the reference and variant alleles of a SNP in a given genomic context. Using ChIP-seq data for FOXA1 they are able to measure the affinity of FOXA1 for a given DNA sequence. They demonstrate that FOXA1 has a high affinity for DNA with a forkhead motif comprising of a $\mathrm{C}$ at position 8 combined with an $\mathrm{A}$ at position 6 , a sequence combination which has six times greater affinity than when the base at position 6 is a G. This emphasizes the concept that transcription factor binding is not a binary event and that quantitative differences in binding can have a profound effect on activity. In the example provided, a base pair change at position 8 of the FKH motif occurs $18 \mathrm{~kb}$ upstream of the TOX3 gene with the variant SNP causing an increase in FOXA1 binding and a concomitant decrease in expression of TOX3, leading to increased cell proliferation.

Another report from the Lupien lab (Zhang et al., 2012) shows functional analysis of a SNP present in a prostate cancer risk locus which changes the tenth position of the FKH DNA motif. Using an in vitro reporter assay they show a 2 -fold decrease in FOXA1 binding to the variant allele compared to the reference allele, resulting in a $50 \%$ increase in luciferase activity. Further supporting this work, a subset of SNPs occurring in hepatocellular carcinoma (HCC) can alter the binding of FOXA2, another forkhead protein, resulting in altered expression, a finding that was confirmed in patient samples on several target genes ( $\mathrm{Li}$ et al., 2012). These examples point to the role that genetic variants can play on FOXA1-DNA interactions and the physiological impact that can have by changing expression levels of key target genes.

\section{FUNCTIONAL RELEVANCE OF FOXA1 ABERRATIONS}

Mutations in the coding sequence of FOXA1 occur in two clusters (Table 1), either in or around the forkhead DNA binding domain or in the C-terminal transactivation domain. Two mutational hotspots are present in the forkhead domain; position D226, mutated in a total of five patients, across both breast and prostate cancer, and position M253, mutated in four prostate tumors. Experimental modeling of these mutations within the DNA binding domain is yet to be conducted therefore it is not known whether they increase or perturb FOXA1 binding. However, previous studies on FOXA1 provide clues as to what may be the downstream effects of these changes.

In breast cancer if these mutations do diminish the binding capability of FOXA1, ER's dependency upon FOXA1 for binding (Hurtado et al., 2011) suggests that these tumors may have evolved to become independent of oestrogen for growth. These tumors would likely be more aggressive as they would not respond 
to standard hormonal therapies (Figure 1B). On the contrary, in prostate cancer, as AR is still able to bind in the absence of FOXA1, a mutation in FOXA1 which inhibits its DNA binding capacity may not inhibit AR binding, but could instead alter its binding profile and transcriptional targets (Sahu et al., 2011; Wang et al., 2011).

Alternatively, mutations within the forkhead domain could affect FOXA1's affinity for its canonical binding site, potentially resulting in binding to new genomic locations, which may result in substantial effects on the transcriptional program within that tumor, for example FOXAl's recruitment to novel sites in the CRPC cell line LNCaP-abl, allows it to become androgen independent (Zhang et al., 2011). FOXA1-DNA associations may also be affected on a site by site basis, due to SNPs occurring in FOXA1 binding sites (Cowper-Sal Lari et al., 2012). Insights into how changes in specific forkhead motifs can affect transcription factor recruitment at that site, as well as downstream gene expression changes, could prove to be essential in determining the "most important" FOXA1 binding events in cancer.

Grasso et al. conducted the first set of functional analysis of the $\mathrm{N}$ - and C-terminal mutations detected, by creating prostate cancer cell lines stably expressing five of the mutants (Grasso et al., 2012). In these preliminary experiments, only one of the mutations, L388M, caused a growth increase over wild type (WT) FOXA1 overexpression. AR-FOXA1 interaction was still important in this mutant cell line as it remained reliant upon androgen for growth. The S453fs mutant and WT FOXA1 were overexpressed in xenograft tumors which resulted in significantly larger tumors, but the mutation posed no growth advantage over WT. Even though the authors themselves express the need for further characterization of the mutations, we could speculate that this cluster of mutations within the C-terminus may change the constituents of the FOXA1 complex. In CRPC, AR splice variants are expressed which could preferentially bind to FOXA1 mutants, a feasible theory as these splice events often occur in the hinge domain of AR, which constitutes its' interaction point with FOXA1 (Wang et al., 2011).

Finally, FOXA1 binding may be increased due to a mutation stabilizing the protein via changes in its' DNA interaction affinity or through copy number gains in the FOXA1 locus resulting in elevated protein levels. In prostate cancer, FOXA1 genomic locus amplification was only observed in metastatic samples (Robbins

\section{REFERENCES}

Badve, S., Turbin, D., Thorat, M. A., Morimiya, A., Nielsen, T. O., Perou, C. M., et al. (2007). FOXA1 expression in breast cancer - correlation with luminal subtype A and survival. Clin. Cancer Res. 13, 4415-4421.

Banerji, S., Cibulskis, K., RangelEscareno, C., Brown, K. K., Carter, S. L., Frederick, A. M., et al. (2012). Sequence analysis of mutations and translocations across breast cancer subtypes. Nature 486, 405-409.

Barbieri, C. E., Baca, S. C., Lawrence, M. S., Demichelis, F., Blattner,
M., Theurillat, J.-P., et al. (2012). Exome sequencing identifies recurrent SPOP, FOXA1 and MED12 mutations in prostate cancer. Nat. Genet. 44, 685-689.

Berger, M. F., Lawrence, M. S., Demichelis, F., Drier, Y., Cibulskis, K., Sivachenko, A. Y., et al. (2011). The genomic complexity of primary human prostate cancer. Nature 470 , 214-220.

Bernardo, G. M., Lozada, K. L., Miedler, J. D., Harburg, G., Hewitt, S. C., Mosley, J. D., et al. (2010). FOXA1 is an essential determinant of $\mathrm{ER} \alpha$ expression and mammary ductal

et al., 2011; Grasso et al., 2012), which correlates with the observation of elevated FOXA1 staining in metastases and high grade tumors (Gerhardt et al., 2012). Increased levels of WT FOXA1 significantly increase prostate cancer proliferation and the size of xenograft tumors (Grasso et al., 2012), possibly due to increased AR-mediated growth. In breast cancer, however, high levels of FOXA1 are a marker of good prognosis (Badve et al., 2007; Hisamatsu et al., 2012) possibly because elevated FOXA1 in breast cancer engenders a greater reliance upon ligand-dependent ER activity to drive tumorigenesis, which in turn would generate hypersensitivity to anti-oestrogen therapies. This theory suggests that breast tumors with amplification of FOXA1 would be more responsive to treatment and the patients would have a better prognosis (Figure 1B).

Reliance upon ligands for nuclear receptor activation could be a fundamental difference between breast and prostate cancer and may explain why the two hormone receptors respond differently to gain and loss of FOXA1. If AR driven prostate cancers readily acquire ligand independence, elevated FOXA1-AR activity would be an advantage to the tumor, supporting the observation that high FOXA1 is a marker of poor patient outcome. In contrast, breast cancers may retain ligand dependence for longer, permitting the paradoxical acquisition of both elevated estrogenmediated ER-FOXA1 activity and increased sensitivity to drugs that block this pathway. It will be critical to determine the key ER and AR target gene changes at high and low FOXA1 levels and any differences in the associated AR/ER protein complexes.

\section{CONCLUSION}

It is evident that FOXA1 can be regulated at a genomic level either through somatic mutations, genomic amplification, or changes to the sequence within binding regions. Due to the lack of current follow up and low frequency of events there is no current indication on how these changes affect patient outcome. As such, it is important to collect functional data on the genomic changes observed in breast and prostate cancer.

\section{ACKNOWLEDGMENTS}

We would like to thank Aisling Redmond and Aurelien Serandour for critical reading. We would like to acknowledge the support of University of Cambridge, Cancer Research UK and Breast Cancer Campaign.

morphogenesis. Development 137 2045-2054.

Carroll, J. S., Meyer, C. A., Song, J., Li, W., Geistlinger, T. R., Eeckhoute, J., et al. (2006). Genome-wide analysis of estrogen receptor binding sites. Nat. Genet. 38, 1289-1297.

Cirillo, L. A., Lin, F. R., Cuesta, I., Friedman, D., Jarnik, M., and Zaret, K. S. (2002). Opening of compacted chromatin by early developmental transcription factors HNF3 (FoxA) and GATA-4. Mol. Cell 9, 279-289.

Cirillo, L. A., McPherson, C. E., Bossard, P., Stevens, K., Cherian, S.,
Shim, E. Y., et al. (1998). Binding of the winged-helix transcription factor HNF3 to a linker histone site on the nucleosome. EMBO J. 17, 244-254.

Clark, K. L., Halay, E. D., Lai, E., and Burley, S. K. (1993). Co-crystal structure of the HNF-3/fork head DNA-recognition motif resembles histone H5. Nature 364, 412-420.

Cowper-Sal Lari, R., Zhang, X., Wright, J. B., Bailey, S. D., Cole, M. D., Eeckhoute, J., et al. (2012). Breast cancer risk-associated SNPs modulate the affinity of chromatin for 
FOXA1 and alter gene expression. Nat. Genet. 44, 1191-1198.

Curtis, C., Shah, S. P., Chin, S.-F., Turashvili, G., Rueda, O. M., Dunning, M. J., et al. (2012). The genomic and transcriptomic architecture of 2,000 breast tumours reveals novel subgroups. Nature 486, 346-352.

Dees, N. D., Zhang, Q., Kandoth, C., Wendl, M. C., Schierding, W., Koboldt, D. C., et al. (2012). MuSiC: identifying mutational significance in cancer genomes. Genome Res. 22, 1589-1598.

Deutsch, L., Wrage, M., Koops, S., Glatzel, M., Uzunoglu, F. G., Kutup, A., et al. (2012). Opposite roles of FOXA1 and NKX2-1 in lung cancer progression. Genes Chromosomes Cancer 51, 618-629.

Ellis, M. J., Ding, L., Shen, D., Luo, J., Suman, V. J., Wallis, J. W., et al. (2012). Whole-genome analysis informs breast cancer response to aromatase inhibition. Nature 486 , 353-360.

Ferlay, J., Shin, H.-R., Bray, F., Forman, D., Mathers, C., and Parkin, D. M. (2010). Estimates of worldwide burden of cancer in 2008: GLOBOCAN (2008). Int. J. Cancer 127, 2893-2917.

Gao, N., Ishii, K., Mirosevich, J., Kuwajima, S., Oppenheimer, S. R., Roberts, R. L., et al. (2005). Forkhead box A1 regulates prostate ductal morphogenesis and promotes epithelial cell maturation. Development 132, 3431-3443.

Gao, N., Zhang, J., Rao, M. A., Case, T. C., Mirosevich, J., Wang, Y., et al. (2003). The role of hepatocyte nuclear factor-3a (Forkhead Box A1) and androgen receptor in transcriptional regulation of prostatic genes. Mol. Endocrinol. 17, 1484-1507.

Gerhardt, J., Montani, M., Wild, P., Beer, M., Huber, F., Hermanns, T., et al. (2012). FOXAl promotes tumor progression in prostate cancer and represents a novel hallmark of castrate-resistant prostate cancer. Am. J. Pathol. 180, 848-861.

Grasso, C. S., Wu, Y.-M., Robinson, D. R., Cao, X., Dhanasekaran, S. M., Khan, A. P., et al. (2012). The mutational landscape of lethal castration-resistant prostate cancer. Nature 487, 239-243.

Hannenhalli, S., and Kaestner, K. H. (2009). The evolution of Fox genes and their role in development and disease. Nat. Rev. Genet. 10, 233-240.
Hisamatsu, Y., Tokunaga, E., Yamashita, N., Akiyoshi, S., Okada, S. Nakashima, Y., et al. (2012). Impact of FOXA1 expression on the prognosis of patients with hormone receptor-positive breast cancer. Ann. Surg. Oncol. 19, 1145-1152.

Hurtado, A., Holmes, K. A., Ross-Innes, C. S., Schmidt, D., and Carroll, J. S. (2011). FOXA1 is a key determinant of estrogen receptor function and endocrine response. Nat. Genet. 43, 27-33.

Jain, R. K., Mehta, R. J., Nakshatri, H., Idrees, M. T., and Badve, S. S. (2011). High-level expression of forkhead-box protein A1 in metastatic prostate cancer. Histopathology 58, 766-772.

Jia, L., Berman, B. P., Jariwala, U., Yan, X., Cogan, J. P., Walters, A., et al. (2008). Genomic androgen receptor-occupied regions with different functions, defined by histone acetylation, coregulators and transcriptional capacity. PLoS ONE 3:e3645. doi: 10.1371/journal.pone.0003645

Kumar, A., White, T. A., Mackenzie, A. P., Clegg, N., Lee, C., Dumpit, R. F., et al. (2011). Exome sequencing identifies a spectrum of mutation frequencies in advanced and lethal prostate cancers. Proc. Natl. Acad. Sci. 108, 17087-17092.

Lee, C. S., Friedman, J. R., Fulmer, J. T., and Kaestner, K. H. (2005). The initiation of liver development is dependent on Foxa transcription factors. Nature 435, 944-947.

Ley, T. J., Mardis, E. R., Ding, L., Fulton, B., McLellan, M. D., Chen, K., et al. (2008). DNA sequencing of a cytogenetically normal acute myeloid leukaemia genome. Nature 456, 66-72.

Li, Z., Tuteja, G., Schug, J., and Kaestner, K. H. (2012). Foxal and Foxa2 are essential for sexual dimorphism in liver cancer. Cell 148, 72-83.

Lin, L., Miller, C. T., Contreras, J. I., Prescott, M. S., Dagenais, S. L., Wu, R., et al. (2002). The hepatocyte nuclear factor 3 a gene, HNF3a (FOXA1), on chromosome band $14 \mathrm{q} 13$ is amplified and overexpressed in esophageal and lung adenocarcinomas. Cancer Res. 62, 5273-5279.

Meyerson, M., Gabriel, S., and Getz, G. (2010). Advances in understanding cancer genomes through secondgeneration sequencing. Nat. Rev. Genet. 11, 685-696.
Nucera, C., Eeckhoute, J., Finn, S., Carroll, J. S., Ligon, A. H., Priolo, C., et al. (2009). FOXA1 is a potential oncogene in anaplastic thyroid carcinoma. Clin. Cancer Res. 15, 3680-3689.

Robbins, C. M., Tembe, W. A., Baker, A., Sinari, S., Moses, T. Y., Beckstrom-Sternberg, S., et al. (2011). Copy number and targeted mutational analysis reveals novel somatic events in metastatic prostate tumors. Genome Res. 21, $47-55$.

Robinson, J. L. L., Macarthur, S., Ross-Innes, C. S., Tilley, W. D., Neal, D. E., Mills, I. G., et al. (2011). Androgen receptor driven transcription in molecular apocrine breast cancer is mediated by FoxA1. EMBO J. 30, 3019-3027.

Robyr, D., Gegonne, A., Wolffe, A. P., and Wahli, W. (2000). Determinants of vitellogenin B1 promoter architecture. J. Biol. Chem. 275, 28291-28300.

Ross-Innes, C. S., Stark, R., Teschendorff, A. E., Holmes, K. A., Ali, H. R., Dunning, M. J., et al. (2012). Differential oestrogen receptor binding is associated with clinical outcome in breast cancer. Nature 481, 389-393.

Sahu, B., Laakso, M., Ovaska, K., Mirtti, T., Lundin, J., Rannikko, A., et al. (2011). Dual role of FoxA1 in androgen receptor binding to chromatin, androgen signalling and prostate cancer. EMBO J. 30, 3962-3976.

Shah, S. P., Roth, A., Goya, R., Oloumi, A., Ha, G., Zhao, Y., et al. (2012). The clonal and mutational evolution spectrum of primary triplenegative breast cancers. Nature 486, 395-399.

Stephens, P. J., Tarpey, P. S., Davies, H., Van Loo, P., Greenman, C., Wedge, D. C., et al. (2012). The landscape of cancer genes and mutational processes in breast cancer. Nature 486, 400-404.

Taylor, B. S., Schultz, N., Hieronymus, H., Gopalan, A., Xiao, Y., Carver, B. S., et al. (2010). Integrative genomic profiling of human prostate cancer. Cancer Cell 18, 11-22.

TCGA. (2012). Comprehensive molecular portraits of human breast tumours. Nature 490, 61-70.

Wan, H., Kaestner, K. H., Ang, S.-L., Ikegami, M., Finkelman, F. D., Stahlman, M. T., et al. (2004). Foxa2 regulates alveolarization and goblet cell hyperplasia. Development 131, 953-964.
Wang, D., Garcia-Bassets, I., Benner, C., Li, W., Su, X., Zhou, Y., et al. (2011). Reprogramming transcription by distinct classes of enhancers functionally defined by eRNA. Nature 474, 390-394.

Wang, Q., Li, W., Liu, X. S., Carroll, J. S., Janne, O. A., Keeton, E. K., et al. (2007). A hierarchical network of transcription factors governs androgen receptor-dependent prostate cancer growth. Mol. Cell 27 , 380-392.

Yasui, K., Imoto, I., Fukuda, Y., Pimkhaokham, A., Yang, Z.-Q., Naruto, T., et al. (2001). Identification of target genes within an amplicon at 14q12-q13 in esophageal squamous cell carcinoma. Genes Chromosomes Cancer $32,112-118$.

Zhang, C., Wang, L., Wu, D., Chen, H., Chen, Z., Thomas-Ahner, J. M., et al. (2011). Definition of a FoxAl Cistrome that is crucial for G1 to Sphase cell-cycle transit in castrationresistant prostate cancer. Cancer Res. 71, 6738-6748.

Zhang, X., Cowper-Sala Lari, R., Bailey, S. D., Moore, J. H., and Lupien, M. (2012). Integrative functional genomics identifies an enhancer looping to the SOX9 gene disrupted by the $17 \mathrm{q} 24.3$ prostate cancer risk locus. Genome Res. 22, 1437-1446.

Conflict of Interest Statement: The authors declare that the research was conducted in the absence of any commercial or financial relationships that could be construed as a potential conflict of interest.

Received: 30 November 2012; paper pending published: 13 December 2012; accepted: 25 January 2013; published online: 14 February 2013.

Citation: Robinson JLL, Holmes KA and Carroll JS (2013) FOXA1 mutations in hormone-dependent cancers. Front. Oncol. 3:20. doi: 10.3389/fonc. 2013.00020

This article was submitted to Frontiers in Molecular and Cellular Oncology, a specialty of Frontiers in Oncology.

Copyright (c) 2013 Robinson, Holmes and Carroll. This is an open-access article distributed under the terms of the Creative Commons Attribution License, which permits use, distribution and reproduction in other forums, provided the original authors and source are credited and subject to any copyright notices concerning any third-party graphics etc. 